\title{
Análisis de la relación \\ entre la gestión del conocimiento y la gestión de la calidad en \\ la empresa Districarnazas Luna*
}

\section{Analysis of the relationship between knowledge management and quality management in the company Districarnazas Luna}

Recibido: 18 de febrero de 2013

Revisado: 15 de marzo de 2013

Aceptado: 27 de mayo de 2013

Andrés Mauricio Díaz Quintero**

Universidad Agraria de Colombia

\section{RESUMEN}

En la perspectiva de conocer las relaciones que han establecido las organizaciones empresariales entre los sistemas de gestión de calidad (SGC) y los modelos de gestión del conocimiento (GC), surge el interés por analizar el caso de Districarnazas Luna, una empresa dedicada a la fabricación y comercialización de pieles curtidas de ganado vacuno.

Los referentes teóricos que sustentan el trabajo se sitúan en los desarrollos respecto a cómo se concibe el conocimiento en las organizaciones, así como las perspectivas, enfoques, modelos y componentes de la gestión de calidad y de la gestión de conocimiento.

Districarnazas Luna ha construido un acervo importante de conocimiento, incorporando nuevas tecnologías y expandiéndose de manera significativa; sin embargo, ello no responde a una dinámica de construcción y transformación del conocimiento intencionada, puesto que se da a partir de las necesidades del negocio, por exigencias del mercado, y no propiamente como consecuencia de los planes estratégicos por mantenerse en una posición de ventaja competitiva, por lo que se resalta la necesidad de diseñar

* Artículo de investigación aplicada

** Correspondencia: Andrés Mauricio Díaz Quintero. Magíster en Calidad y Gestión Integral. Director del programa de Ingeniería Industrial de la Universidad Agraria de Colombia. Correo electrónico: andresmauriciod@yahoo.com.mx.

Trabajo de grado para optar al título de Magister en Calidad y Gestión Integral, dirigido por Mg. Álvaro Perdomo Burgos. 
estrategias para movilizar la asimilación y realización de prácticas encaminadas a generar una cultura de conocimiento en la compañía. Se analizó lo que ocurre en la organización y se esquematizó un modelo que le apunta a la articulación entre la gestión de la calidad con la gestión del conocimiento.

Palabras clave: capital intelectual, gestión de la calidad, gestión del conocimiento.

\section{ABSTRACT}

On the prospect of knowing the relationships that have been established by entrepreneurial organizations between quality management systems (QMS) and knowledge management systems (KMS), arises the interest in analyzing the case of Districarnazas Luna, a company dedicate to the production and commercialization of cattle tanned hides.

Theoretical frameworks that support the work lie on the developments regarding how knowlege is conceived in the organizations, as well as the perspectives, approaches, models and components of quality management and knowldge management.

Districarnazas Luna has built an important wealth of knowledge, incorporating new technologies and expanding significantly; however, that does not respond to an intentional dynamic of building and transforming knowledge, since it occurs from the needs of the business, demands of the market, and not properly as a result of strategic plans to maintain a position of competitive advantage, which is why it is stressed the need to design strategies to move the uptake and implementation of practices aimed at creating a culture of knowledge in the company. It was analyzed what occurs in the organization and a model was outlined which aims at the articulation between quality management and knowledge management.
Keywords: Intellectual capital, quality management, knowledge management.

\section{INTRODUCCIÓN}

Para muchas empresas resulta esencial hoy en día incrementar el conocimiento de sus trabajadores como estrategia para mejorar el desempeño organizacional y ubicarse en ámbitos altamente competitivos; ello, de cara a sus intereses comerciales, pero también a las necesidades emergentes que les demandan nuevas maneras de penetrar y mantenerse en el mercado, así como para realizar transformaciones o ajustes endógenos que contribuyan al mejoramiento de las relaciones interpersonales, al clima organizacional y a la comunicación del talento humano con el cual cuentan.

En este contexto, la gestión del conocimiento se configura en una estrategia que puede potenciar el funcionamiento del sistema de gestión de la calidad (SGC) en la empresa Districarnazas Luna, de manera tal que se propenda por el conocimiento y el aprendizaje como dinamizadores de los objetivos organizacionales, pero también por la cualificación de sus trabajadores, aspectos que muy posiblemente revertirán en procesos de desarrollo e innovación que se cristalicen en ventajas competitivas para la organización frente a los vaivenes del mercado; igualmente, problemas, como las ineficiencias relacionadas con el clima organizacional, encontrarán respuesta con la articulación entre un modelo que gestione el conocimiento y un sistema de gestión de calidad.

Districarnazas Luna tiene una experiencia de 20 años en la fabricación y comercialización de pieles curtidas de ganado vacuno. Actualmente, cuenta con una planta de 82 trabajadores y está ubicada en el barrio La Coruña; allí se hace el proceso de pelambre y alistamiento para el curtido y transformación de la piel animal en cuero para diferentes usos. Además, se procesa 
la piel, que consiste en el curtido, curado, tinturado, alistamiento y demás procesos derivados.

Una de las estrategias más importantes a través del tiempo ha sido la definición clara de su mercado, lo que le ha generado grandes posibilidades de posicionamiento, crecimiento e incorporación de tecnología a sus procesos. Por ejemplo, desde hace más de diez años, Districarnazas Luna le provee cuero al Ministerio de Defensa Nacional como parte de su material de campaña. Esto obligó a la empresa a certificarse bajo el modelo ISO 9000:2000, como exigencia para participar en las diferentes licitaciones.

La empresa plantea como política de calidad reflejar idoneidad en el diseño y procesamiento de pieles para la fabricación de cueros con destino a las industrias de calzado, marroquinería, tapicería y prendas de vestir, aplicando valores humanos, con compromiso de mejorar continuamente y satisfacer las necesidades del cliente, cumpliendo con los requisitos de orden legal y entregando rentabilidad con el fin de brindar confianza y estimular reinversión.

En este escenario, resultó preponderante preguntarse de qué manera se desarrolla e implementa la gestión del conocimiento (GC) en la empresa Districarnazas Luna a partir de la certificación en la norma ISO 9001.

Para tal efecto, se trazó como objetivo general evaluar la relación entre la GC y el SGC de Districarnazas Luna y a partir de lo detectado proponer un esquema que permita articular el modelo de gestión de la calidad con el modelo de gestión del conocimiento.

Como objetivos específicos se encuentran: aportar a la discusión sobre la relación entre el SGC y la GC que se ha desarrollado en el sector académico responsable de construir marcos referenciales para este debate; establecer la manera cómo la empresa Districarnazas Luna fundamenta la estructura del SGC acorde con la teoría del capital intelectual, y determinar cómo, a partir de la implementación de sistemas de gestión de calidad, se puede incorporar la gestión del conocimiento en la empresa Districarnazas Luna.

Con esta lógica, la investigación se soporta en los desarrollos conceptuales que se han construido con relación a los SGC y GC.

\section{METODOLOGÍA}

El estudio es de carácter descriptivo, en la medida en que pretende dar claridad conceptual y metodológica acerca de los dos modelos que estructuran el objeto de investigación; de la misma manera, se aborda un tipo de investigación exploratoria, dado que indaga en la empresa Districarnazas Luna la existencia de algún tipo de articulación entre la gestión del conocimiento y la gestión de la calidad.

La investigación se estructuró en los siguientes momentos:

1. Análisis documental de la información relacionada con los modelos teóricos sobre gestión del conocimiento y gestión de la calidad.

2. Construcción de un sistema categorial preliminar que profundiza en el objeto de estudio y entiende el proceso de implementación de un modelo de gestión del conocimiento y su articulación con el modelo de calidad existente en la empresa Districarnazas Luna.

3. Elaboración de una encuesta cuyo objetivo fue indagar entre trabajadores y directivos de la empresa el grado de madurez de la gestión del conocimiento. Las preguntas surgen del sistema preliminar de categorías. 
4. Se hizo un muestreo estadístico a fin de calcular la muestra a la que se debe aplicar el instrumento de investigación.

5. Desarrollo del estudio de campo; en él se aplican 25 encuestas a trabajadores del área de producción y operaciones, cinco al personal administrativo y a cinco jefes y directivos de la empresa, a fin de obtener información acerca de la temática que aborda esta investigación.

6. Tabulación y análisis de los datos, resultado del trabajo de campo; en esta etapa se procedió a sistematizar e interpretar los resultados de manera analítica.

7. Análisis y contrastación de las observaciones que arrojaron las encuestas y el cruce de esta información con los modelos de gestión de calidad y gestión del conocimiento, a fin de presentar una propuesta para implementar un esquema que permita a Districarnazas Luna gestionar el conocimiento, lo cual se hace a partir de matrices categoriales.

\section{RESULTADOS Y DISGUSIÓN}

Los principales resultados de la investigación fueron:

\section{Diagnóstico del nivel de madurez de la empresa Districarnazas Luna frente a la gestión del conocimiento}

Del anáisis documental se tomó como referencia la herramienta de autoevaluación contenida en la norma ISO 9004, a partir de la cual se definieron las categorías preliminares y se adapta un instrumento que el investigador ajusta a modo de matriz. Este se categoriza de acuerdo con los elementos que se consideran relevantes en el desarrollo de esta investigación y que atienden algunas características de los modelos teóricos seleccionados (teoría del capital intelectual).
Establecer el grado de madurez de la empresa proporcionará una clara orientación del desempeño de sus procesos, del grado de participación de sus trabajadores y de la apropiación de ellos frente a los valores y estrategias corporativas; igualmente, dará pautas para identificar oportunidades de mejora que conduzcan a la innovación, el aprendizaje continuo y las prácticas dirigidas hacia la gestión del conocimiento.

La matriz contempla categorías de análisis, elementos clave que se constituyen en referentes de evaluación de los procesos dinamizados en Districarnazas Luna en relación con la GC. Se indica también el número de pregunta que corresponde a ese elemento clave y, finalmente, se incluyen los niveles de madurez que frente a la gestión del conocimiento ha alcanzado la empresa.

La muestra del personal de Districarnazas Luna, a la cual se aplicará la encuesta, se determina mediante muestreo probabilístico de la siguiente forma:

1. El total de trabajadores de la empresa es de 82 , la muestra calculada arrojó un número de 35 trabajadores que se encuestaron y entrevistaron. Esto se obtuvo con la siguiente fórmula estadística:

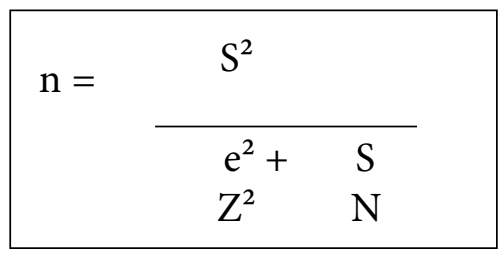

Donde:

$\mathrm{n}=$ tamaño de la muestra.

$S=$ desviación estándar.

$\mathrm{e}=$ error 0 diferencia máxima entre la media muestral y la media poblacional que se está en disposición de aceptar, con el nivel de confianza definido.

$\mathrm{Z}$ = número de desviaciones estándar que producirá el nivel de confianza deseada.

$\mathrm{N}=$ población. 
Para este estudio se toma lo siguiente:

El nivel de confianza requerido es del $95 \%$, por tanto $Z=1,96$, con un error en el estudio del $5 \%$ y una desviación estándar de 0,20 . La población es de 82 trabajadores; al aplicar la fórmula se obtuvo lo siguiente:

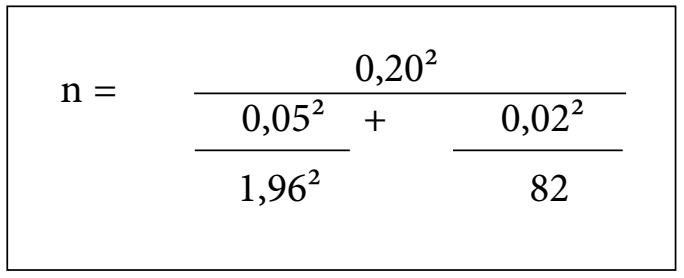

$\mathrm{n}=35$ encuestas por aplicar.

La matriz de la cual surge el cuestionario de la encuesta y la escala de evaluación se presenta en la tabla 1.

Tabla 1. Matriz de evaluación del grado de madurez de la empresa

Districarnazas Luna frente a la gestión del conocimiento

\begin{tabular}{|c|c|c|c|c|c|c|}
\hline \multirow[b]{2}{*}{ CATEGORÍAS } & \multirow{2}{*}{$\begin{array}{l}\text { ELEMENTOS } \\
\text { CLAVE } \\
\text { (VARIABLES) }\end{array}$} & \multirow{2}{*}{$\begin{array}{l}\text { PREGUNTAS } \\
\text { EN LAS } \\
\text { ENCUESTAS }\end{array}$} & \multicolumn{4}{|c|}{ NIVEL DE MADUREZ DE LA EMPRESA } \\
\hline & & & NIVEL 1 & NIVEL 2 & NIVEL 3 & NIVEL 4 \\
\hline $\begin{array}{c}\text { ESTRATEGIA } \\
\text { DE LA } \\
\text { ORGANIZACIÓN }\end{array}$ & $\begin{array}{l}\text { Divulgación } \\
\text { de la } \\
\text { información, } \\
\text { misión, visión, } \\
\text { objetivos, } \\
\text { políticas } \\
\text { de participación } \\
\text { de los } \\
\text { trabajadores }\end{array}$ & $\begin{array}{l}\mathrm{A} 1, \mathrm{~A} 2, \mathrm{~B} 1, \mathrm{~B} 2, \\
\mathrm{~B} 23, \mathrm{~B} 26\end{array}$ & $\begin{array}{l}\text { La organización } \\
\text { se limita a } \\
\text { divulgar entre } \\
\text { sus trabajadores } \\
\text { las políticas, } \\
\text { misión, visión, } \\
\text { objetivos, sin } \\
\text { retroalimentar } \\
\text { entre ellos } \\
\text { el nivel de } \\
\text { percepción e } \\
\text { importancia. }\end{array}$ & $\begin{array}{l}\text { Se propician } \\
\text { encuentros } \\
\text { para socializar } \\
\text { de manera } \\
\text { continua sus } \\
\text { estrategias } \\
\text { corporativas } \\
\text { mediante } \\
\text { la difusión } \\
\text { de las políticas, } \\
\text { misión, visión, } \\
\text { objetivos. }\end{array}$ & $\begin{array}{l}\text { Se comunica } \\
\text { e involucra a } \\
\text { sus trabajadores } \\
\text { en la definición } \\
\text { de la estrategia } \\
\text { y la política } \\
\text { corporativa; } \\
\text { se evalúa } \\
\text { el compromiso } \\
\text { del trabajador } \\
\text { en ellas. }\end{array}$ & $\begin{array}{l}\text { La organización } \\
\text { comunica } \\
\text { oportuna } \\
\text { y periódicamente } \\
\text { la estrategia y } \\
\text { política y genera } \\
\text { mecanismos para } \\
\text { su apropiación, } \\
\text { aceptación y } \\
\text { apoyo por parte } \\
\text { de los } \\
\text { trabajadores; } \\
\text { se hace un } \\
\text { seguimiento } \\
\text { continuo de esto. }\end{array}$ \\
\hline \multirow{4}{*}{$\begin{array}{c}\text { GESTIÓN } \\
\text { PARA EL ÉXITO } \\
\text { SOSTENIDO } \\
\text { DE LA } \\
\text { ORGANIZACIÓN }\end{array}$} & $\begin{array}{l}\text { Productos y } \\
\text { procesos exitosos }\end{array}$ & $\mathrm{A} 17, \mathrm{~A} 21, \mathrm{~B} 24$ & \multirow{4}{*}{$\begin{array}{l}\text { El sistema } \\
\text { de gestión de } \\
\text { calidad está } \\
\text { basado en los } \\
\text { procedimientos } \\
\text { y su éxito radica } \\
\text { en la aceptación } \\
\text { del producto por } \\
\text { parte del cliente. } \\
\text { Da respuesta } \\
\text { a los cambios } \\
\text { o necesidades } \\
\text { del momento. }\end{array}$} & \multirow{4}{*}{$\begin{array}{l}\text { El éxito de la } \\
\text { organización se } \\
\text { define cuando } \\
\text { la empresa } \\
\text { planifica } \\
\text { el desarrollo } \\
\text { de sus productos } \\
\text { y procesos, } \\
\text { el SGC se basa } \\
\text { en procesos, se } \\
\text { tiene en cuenta } \\
\text { la competencia } \\
\text { para comparar } \\
\text { productos. }\end{array}$} & \multirow{4}{*}{$\begin{array}{l}\text { La base } \\
\text { de su éxito } \\
\text { se da en } \\
\text { la gestión } \\
\text { por procesos, } \\
\text { tiene en cuenta } \\
\text { los requisitos } \\
\text { de las partes } \\
\text { interesadas para } \\
\text { la producción } \\
\text { de sus bienes. }\end{array}$} & \multirow{4}{*}{$\begin{array}{l}\text { La organización } \\
\text { satisface de } \\
\text { manera coherente } \\
\text { las partes } \\
\text { interesadas en } \\
\text { el largo plazo, hace } \\
\text { un seguimiento } \\
\text { permanente del } \\
\text { entorno, evalúa } \\
\text { los impactos } \\
\text { individuales sobre } \\
\text { el desempeño } \\
\text { de esta, identifica } \\
\text { riesgos asociados } \\
\text { a corto y largo } \\
\text { plazo. }\end{array}$} \\
\hline & SGG & A19, B21, & & & & \\
\hline & $\begin{array}{l}\text { Entorno de } \\
\text { la organización } \\
\text { Benchmarking }\end{array}$ & B17 & & & & \\
\hline & $\begin{array}{l}\text { Partes } \\
\text { interesadas }\end{array}$ & B17 & & & & \\
\hline
\end{tabular}




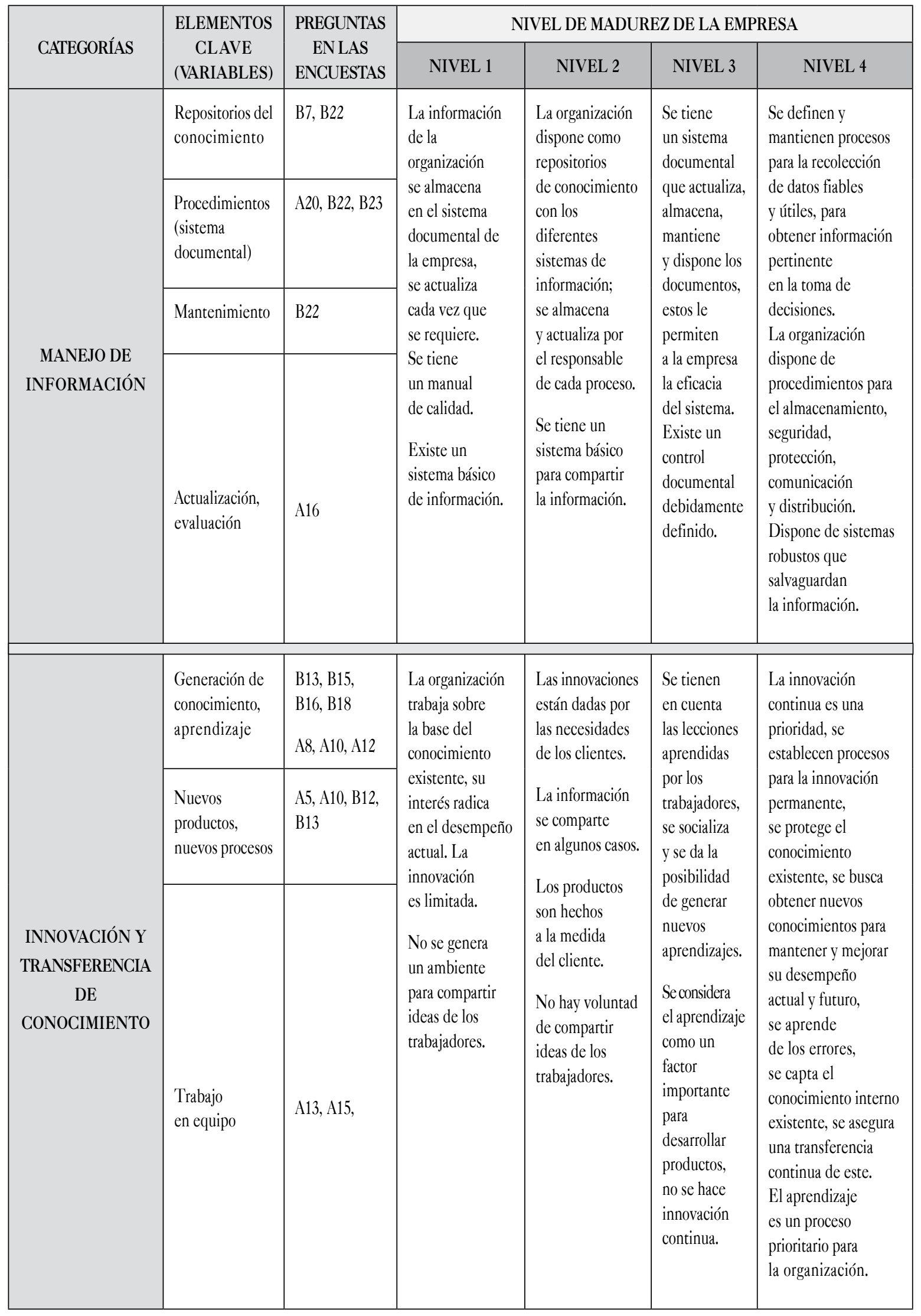




\begin{tabular}{|c|c|c|c|c|c|c|}
\hline \multirow{2}{*}{ CATEGORÍAS } & \multirow{2}{*}{$\begin{array}{c}\text { ELEMENTOS } \\
\text { CLAVE } \\
\text { (VARIABLES) }\end{array}$} & \multirow{2}{*}{$\begin{array}{c}\text { PREGUNTAS } \\
\text { EN LAS } \\
\text { ENCUESTAS }\end{array}$} & \multicolumn{4}{|c|}{ NIVEL DE MADUREZ DE LA EMPRESA } \\
\hline & & & NIVEL 1 & NIVEL 2 & NIVEL 3 & NIVEL 4 \\
\hline $\begin{array}{c}\text { EVALUACIÓN } \\
\text { DEL } \\
\text { DESEMPEÑO }\end{array}$ & $\begin{array}{l}\text { Desempeño } \\
\text { del trabajador }\end{array}$ & $\begin{array}{l}\text { B8, B10, B11 } \\
\text { A5 }\end{array}$ & $\begin{array}{l}\text { La organización } \\
\text { evalúa el desempeño } \\
\text { con el propósito } \\
\text { de tomar decisiones } \\
\text { frente a la mejora } \\
\text { de algún proceso, } \\
\text { que propicie cambios } \\
\text { para evitar errores } \\
\text { que afecten al } \\
\text { cliente. }\end{array}$ & $\begin{array}{l}\text { Se considera } \\
\text { importante } \\
\text { evaluar el } \\
\text { desempeño } \\
\text { del trabajador } \\
\text { a fin de buscar } \\
\text { la mejora de su } \\
\text { competencia } \\
\text { laboral. }\end{array}$ & $\begin{array}{l}\text { Se evalúa el } \\
\text { desempeño del } \\
\text { trabajador con el } \\
\text { fin de propiciar } \\
\text { una cultura de } \\
\text { mejora, que } \\
\text { fortalezca las } \\
\text { competencias } \\
\text { laborales } \\
\text { mediante una } \\
\text { política de } \\
\text { capacitación y } \\
\text { entrenamiento. }\end{array}$ & $\begin{array}{l}\text { Se busca aprender de } \\
\text { los errores, a fin de } \\
\text { determinar factores } \\
\text { clave de éxito. Se } \\
\text { busca identificar } \\
\text { limitaciones de } \\
\text { los individuos en } \\
\text { su desempeño, se } \\
\text { busca la oportunidad } \\
\text { para aumentar } \\
\text { la competencia } \\
\text { laboral de los } \\
\text { trabajadores. }\end{array}$ \\
\hline $\begin{array}{c}\text { USO DE } \\
\text { TECNOLOGÍA }\end{array}$ & $\begin{array}{l}\text { Conocimientos, } \\
\text { información, } \\
\text { TIC }\end{array}$ & $\begin{array}{l}\text { A18, B19, } \\
\text { B20 }\end{array}$ & $\begin{array}{l}\text { Se utilizan las } \\
\text { tecnologías básicas } \\
\text { de la información } \\
\text { para mejorar } \\
\text { el desempeño del } \\
\text { proceso y cumplir } \\
\text { con el estándar } \\
\text { de los productos. } \\
\text { No hay políticas } \\
\text { de actualización de } \\
\text { tecnología. }\end{array}$ & $\begin{array}{l}\text { Se implementan } \\
\text { tecnologías a fin } \\
\text { de mejorar los } \\
\text { conocimientos } \\
\text { y generar mayor } \\
\text { aprendizaje } \\
\text { entre los } \\
\text { trabajadores. } \\
\text { Se actualiza } \\
\text { tecnología } \\
\text { en casos } \\
\text { extremadamente } \\
\text { necesarios. }\end{array}$ & $\begin{array}{l}\text { La tecnología } \\
\text { está dispuesta } \\
\text { para todas } \\
\text { las partes } \\
\text { interesadas. } \\
\text { Se renueva cada } \\
\text { vez que se crea } \\
\text { necesario. }\end{array}$ & $\begin{array}{l}\text { La tecnología es un } \\
\text { mecanismo con el cual } \\
\text { se busca aumentar } \\
\text { el desempeño en los } \\
\text { procesos estratégicos. } \\
\text { Se propicia la } \\
\text { actualización } \\
\text { permanente de } \\
\text { tecnología. El uso } \\
\text { de las tecnologías de } \\
\text { la información busca } \\
\text { facilitar el aprendizaje } \\
\text { y conocimiento } \\
\text { continuo en la } \\
\text { organización. }\end{array}$ \\
\hline \multirow{5}{*}{$\begin{array}{c}\text { PERSONAS } \\
\text { ENLA } \\
\text { ORGANIZACIÓN }\end{array}$} & Incentivos & A14 & \multirow{5}{*}{$\begin{array}{l}\text { No hay programas } \\
\text { de incentivos } \\
\text { que estimulen } \\
\text { la generación de } \\
\text { ideas en beneficio } \\
\text { de los procesos. } \\
\text { Las personas } \\
\text { se consideran } \\
\text { recursos, no tienen } \\
\text { mayor incidencia } \\
\text { con los objetivos } \\
\text { estratégicos de la } \\
\text { empresa. Se adolece } \\
\text { de un plan de carrera } \\
\text { que les permita a } \\
\text { los trabajadores el } \\
\text { desarrollo permanente. } \\
\text { No hay políticas } \\
\text { definidas respecto } \\
\text { a la formación } \\
\text { de los trabajadores, } \\
\text { las competencias } \\
\text { tienen una } \\
\text { importancia menor. }\end{array}$} & \multirow{5}{*}{$\begin{array}{l}\text { Las personas } \\
\text { también se } \\
\text { consideran } \\
\text { como recurso, } \\
\text { con algunos } \\
\text { objetivos } \\
\text { asignados, } \\
\text { los procesos } \\
\text { de capacitación } \\
\text { se dan de } \\
\text { manera } \\
\text { eventual. } \\
\text { Las competencias } \\
\text { laborales } \\
\text { tienen algún } \\
\text { significado, } \\
\text { se busca } \\
\text { propiciar un } \\
\text { mecanismo de } \\
\text { adiestramiento } \\
\text { por medio de las } \\
\text { competencias. }\end{array}$} & \multirow{5}{*}{$\begin{array}{l}\text { La organización } \\
\text { promueve la } \\
\text { formación de } \\
\text { redes internas } \\
\text { que permiten } \\
\text { el aprendizaje } \\
\text { colectivo y la } \\
\text { socialización del } \\
\text { conocimiento. } \\
\text { Los individuos } \\
\text { conocen sus } \\
\text { competencias } \\
\text { y la manera } \\
\text { cómo estas } \\
\text { contribuyen } \\
\text { a la empresa. }\end{array}$} & \multirow{5}{*}{$\begin{array}{l}\text { La organización } \\
\text { apoya la construcción } \\
\text { de redes internas } \\
\text { y externas, en las } \\
\text { que participan todos } \\
\text { los trabajadores } \\
\text { de la compañía. } \\
\text { Mediante ellas, se } \\
\text { genera y transfiere } \\
\text { conocimiento. } \\
\text { Existen planes } \\
\text { definidos de carrera, } \\
\text { el trabajador tiene } \\
\text { una mayor movilidad } \\
\text { dentro de la empresa, } \\
\text { la capacitación está } \\
\text { totalmente ligada } \\
\text { a la estrategia } \\
\text { organizacional, } \\
\text { se tiene definido } \\
\text { el reconocimiento } \\
\text { a los individuos. }\end{array}$} \\
\hline & $\begin{array}{l}\text { Clima para } \\
\text { la creación } \\
\text { de ideas }\end{array}$ & A11 & & & & \\
\hline & $\begin{array}{l}\text { Planes } \\
\text { de carrera }\end{array}$ & B9 & & & & \\
\hline & Capacitación & $\begin{array}{l}\text { A6, A7, A9, } \\
\text { B3, B4, B5, } \\
\text { B6, B7 }\end{array}$ & & & & \\
\hline & $\begin{array}{l}\text { Competencias } \\
\text { laborales }\end{array}$ & $\begin{array}{l}\mathrm{A} 3, \mathrm{~A} 4, \mathrm{~A} 22, \\
\mathrm{~B} 25\end{array}$ & & & & \\
\hline
\end{tabular}

Fuente: Adaptado de la Norma NTC ISO 9004 por el autor de la investigación. 
Tabla 2. Tabla de valoración

\begin{tabular}{|c|c|c|c|}
\hline \multicolumn{2}{|c|}{$\begin{array}{c}\text { NIVEL DE } \\
\text { MADUREZ }\end{array}$} & IDENTIFICACIÓN & PUNTUACIÓN \\
\hline 1 & bajo & & 1 \\
\hline 2 & medio & & 2 \\
\hline 3 & alto & & 3 \\
\hline 4 & muy alto & & 4 \\
\hline
\end{tabular}

Escala de valoración:

$0-20 \%$ muy bajo

$21-40 \%$ bajo

$41-60 \%$ medio

$61-80 \%$ alto

$81-100 \%$ muy alto

\section{Evaluación y resultados de la matriz de madurez de la empresa Districarnazas Luna}

- Estrategia de la organización. La empresa muestra falencias en cuanto a su estrategia, en tanto que allí no se promueve una participación activa de los trabajadores, se limita únicamente a la divulgación sin ninguna cohesión ni articulación con la internalización de las partes interesadas, por eso se considera en un nivel bajo (tabla 3).

- Gestión para el éxito sostenido de la organización. Districarnazas Luna tiene algunas prácticas que le permiten ser una empresa exitosa en el mercado, tiene claras las actividades que son consideradas clave para el éxito del negocio. No obstante, su SGC no es fuerte, no está bien consolidado, basa su éxito en las mejores prácticas de sus competidores. Se considera un nivel medio, dado que sus prácticas no responden de una manera coherente con el sistema de calidad (tabla 3).

- Manejo de información. No se utiliza de manera adecuada ni para la capacitación ni para la generación de nuevo conocimiento; la documentación del SGC se utiliza para almacenarlo, no hay una directriz clara frente a su utilización para adquirir nuevas ideas, es muy estático, no hay divulgación del conocimiento ni una idea clara con relación a la actualización de los procedimientos. Se evalúa con nivel bajo (tabla 3).

\section{- Innovación y transferencia de conocimiento. A} pesar de que la innovación se considera importante, no hay políticas que evidencien mecanismos y estímulos a las ideas innovadoras ni al trabajo interdisciplinario que conlleve nuevas propuestas de procesos o nuevos desarrollos. Es bajo el aporte de los empleados en torno a mejoras, no se observa transmisión de conocimientos, salvo de los jefes inmediatos. Sin embargo, la empresa ha hecho algunas innovaciones en los últimos años para estar al día en el uso de tecnologías. Se considera un nivel bajo para este ítem (tabla 3).

- Evaluación del desempeño. A pesar de que la empresa sí evalúa el desempeño, esta no es una actividad considerada para tener en cuenta como lecciones aprendidas de los trabajadores, por ende no hay ninguna relación con su aporte a la gestión del conocimiento. Se evalúa en nivel bajo (tabla 3).

- Uso de tecnología. La empresa posibilita el trabajo de las personas por este medio, la información se sistematiza por medios magnéticos, también se utiliza el SGC para este propósito; sin embargo, no hay políticas claras en cuanto a la actualización. Se evalúa en nivel bajo (tabla 3).

- Personas en la organización. Se siguen manejando como recursos, no existen incentivos que motiven a los individuos a proponer ideas y cambios para mejorar, los procesos de capacitación son aislados y se adolece de un plan de carrera que ayude a potenciar el factor humano y se convierta en una estrategia que le permita a la organización la mejora en el desempeño. Se considera en un nivel bajo (tabla 3). 
Tabla 3. Puntaje por categorías

\begin{tabular}{|l|c|c|c|}
\hline \multicolumn{1}{|c|}{ CATEGORÍA } & PUNTUACIÓN & PUNTAJE POSIBLE & $\%$ \\
\hline Estrategia de la organización & 1 & 4 & 25 \\
\hline Gestión para el éxito sostenido de la organización & 2 & 4 & 50 \\
\hline Manejo de información & 1 & 4 & 25 \\
\hline Innovación y transferencia de conocimiento & 1 & 4 & 25 \\
\hline Evaluación del desempeño & 1 & 4 & 25 \\
\hline Uso de tecnología & 1 & 4 & 25 \\
\hline Personas en la organización & 1 & 4 & 25 \\
\hline Totales & 8 & 28 & 28 \\
\hline
\end{tabular}

La empresa se encuentra en un nivel de madurez bajo respecto a la gestión del conocimiento; ello implica que Districarnazas Luna deberá aplicar un modelo de GG que le permita capitalizar todo el conocimiento que ha construido desde sus inicios, de sus trabajadores y de todas las personas involucradas en el proceso, para conducir a la empresa hacia el éxito sostenido.

\section{Propuesta de implementación de un modelo de gestión del conocimiento para Districarnazas Luna}

Para efectos de esta investigación, es pertinente validar aquellos elementos que se consideran integradores y son comunes en los modelos de calidad y de gestión del conocimiento. Estos se recogen en una matriz categorial, a partir de la cual se fundamenta la propuesta para la empresa objeto de este estudio.

La tabla 4 muestra los elementos comunes de los modelos

de calidad orientados hacia la gestión del conocimiento.

Tabla 4. Matriz resumen de los modelos de calidad

\begin{tabular}{|c|c|c|c|}
\hline MODELO & CRITERIO & $\begin{array}{c}\text { ELEMENTO COMÚN } \\
\text { E INTEGRADOR } \\
\text { EN UN MODELO DE GESTIÓN } \\
\text { DEL CONOCIMIENTO }\end{array}$ & OBJETIVO ESTRATÉGICO \\
\hline \multirow{4}{*}{ Fundibeq } & \multirow{4}{*}{$\begin{array}{l}\text { Innovación } \\
\text { y mejora } \\
\text { continua }\end{array}$} & Tecnologías de información. & Potenciar la utilización de nuevas tecnologías. \\
\hline & & Orientación a personas. & $\begin{array}{l}\text { Aprendizaje con el apoyo del líder. } \\
\text { Participación en grupos de mejora. }\end{array}$ \\
\hline & & Potenciar el talento de las personas. & $\begin{array}{l}\text { Trabajo en equipo, desarrollo de personas, influencia de los } \\
\text { demás. }\end{array}$ \\
\hline & & Información. & $\begin{array}{l}\text { Mejora en la calidad de las bases de datos. } \\
\text { Seguridad y confiabilidad de la información. }\end{array}$ \\
\hline
\end{tabular}


Andrés Mauricio Díaz Quintero

\begin{tabular}{|c|c|c|c|}
\hline MODELO & CRITERIO & $\begin{array}{l}\text { ELEMENTO COMÚN } \\
\text { E INTEGRADOR } \\
\text { EN UN MODELO DE GESTIÓN } \\
\text { DEL CONOCIMIENTO }\end{array}$ & OBJETIVO ESTRATÉGICO \\
\hline $\begin{array}{l}\text { Malcolm } \\
\text { Baldrige }\end{array}$ & $\begin{array}{l}\text { Excelencia en } \\
\text { el desempeño }\end{array}$ & $\begin{array}{l}\text { Medición, análisis y gestión } \\
\text { del conocimiento. }\end{array}$ & $\begin{array}{l}\text { Administración y protección del conocimiento. } \\
\text { Diseño de los sistemas de información, para crear valor } \\
\text { a los clientes. }\end{array}$ \\
\hline EFQM & $\begin{array}{l}\text { Innovación, } \\
\text { el aprendizaje } \\
\text { y la mejora } \\
\text { continua }\end{array}$ & Personas. & $\begin{array}{l}\text { Gestionar y desarrollar los conocimientos de las personas } \\
\text { que la constituyen y liberar todo su potencial, tanto } \\
\text { individualmente como en equipo y en el conjunto } \\
\text { de la organización. }\end{array}$ \\
\hline \multirow{3}{*}{ ISO 9004} & \multirow[t]{3}{*}{$\begin{array}{l}\text { Éxito } \\
\text { sostenido } \\
\text { de las } \\
\text { organizaciones }\end{array}$} & Gestión de las personas. & $\begin{array}{l}\text { Creación de valor a partir de la participación de las personas. } \\
\text { Crecimiento, aprendizaje, transferencia de conocimientos } \\
\text { y trabajo en equipo. } \\
\text { Definir un plan de desarrollo para las personas. } \\
\text { Identificación de competencias. }\end{array}$ \\
\hline & & Innovación y aprendizaje. & $\begin{array}{l}\text { Participación y motivación de los trabajadores. } \\
\text { Mejorar condiciones del entorno para propiciar la innovación. } \\
\text { Revisar las lecciones aprendidas. }\end{array}$ \\
\hline & & $\begin{array}{l}\text { Conocimientos, información } \\
\text { y tecnología. }\end{array}$ & $\begin{array}{l}\text { Establecer procesos para gestionar los conocimientos, información } \\
\text { y tecnología. }\end{array}$ \\
\hline
\end{tabular}

Los elementos comunes e integradores se refieren a aquellos aspectos definidos en cada uno de los modelos de calidad que orientan la gestión de las organizaciones hacia la GC. Dado que uno de los propósitos de esta investigación es articular un modelo de calidad con un modelo de gestión del conocimiento, se presenta a continuación una matriz que sintetiza los fundamentos clave de algunos modelos de gestión del conocimiento para diseñar un enfoque integrador con la calidad. 
Tabla 5. Matriz categorial de elementos comunes e integradores de los modelos de gestión del conocimiento

\begin{tabular}{|c|c|c|c|}
\hline $\begin{array}{c}\text { MODELO } \\
\text { DE GESTIÓN } \\
\text { DEL CONOCIMIENTO }\end{array}$ & CARACTERÍSTICAS PRINCIPALES & $\begin{array}{c}\text { ELEMENTOS } \\
\text { QUE EMERGEN } \\
\text { PROPIOS } \\
\text { DE LA GESTIÓN } \\
\text { DE LA CALIDAD }\end{array}$ & $\begin{array}{c}\text { ESTRATEGIA } \\
\text { DE LA ORGANIZACIÓN }\end{array}$ \\
\hline $\begin{array}{l}\text { Creación del conocimiento - } \\
\text { Nonaka y Takeuchi }\end{array}$ & $\begin{array}{l}\text { El conocimiento tiene tres dimensiones: } \\
\text { 1) hay un gran poder de aprendizaje } \\
\text { por la experiencia directa, basado en prueba } \\
\text { y error; 2) una vez realizado el conocimiento } \\
\text { tácito, de alguna forma se empieza a } \\
\text { pensar en la innovación, visto este como } \\
\text { un proceso altamente individual, personal } \\
\text { y de renovación organizacional; } \\
\text { 3) el conocimiento organizacional no solo } \\
\text { puede adquirirse a través de manuales, } \\
\text { libros o lecturas, y entender que a través } \\
\text { de las metáforas, representaciones o } \\
\text { experiencias se gana la visión, perspicacia } \\
\text { e intuición necesarias en la creación } \\
\text { y acumulación de conocimiento. } \\
\text { El proceso de creación de conocimiento } \\
\text { se realiza a través de una espiral, en la } \\
\text { cual se busca que la organización movilice } \\
\text { y transforme el conocimiento tácito } \\
\text { de los individuos y lo convierta en } \\
\text { conocimiento explícito. }\end{array}$ & $\begin{array}{l}\text { Gestión por procesos } \\
\text { Sistema documental } \\
\text { Gestión de las } \\
\text { personas }\end{array}$ & $\begin{array}{l}\text { La organización debe propiciar } \\
\text { espacios para la generación } \\
\text { de conocimiento y nuevos } \\
\text { aprendizajes, propender } \\
\text { por el trabajo compartido, } \\
\text { aprendizajes colectivos. } \\
\text { Tener repositorios } \\
\text { de conocimiento. }\end{array}$ \\
\hline Modelo KMAT & $\begin{array}{l}\text { El modelo propone cuatro elementos } \\
\text { facilitadores (liderazgo, cultura, } \\
\text { tecnología y medición) que favorecen } \\
\text { el proceso de administrar el conocimiento } \\
\text { organizacional. }\end{array}$ & $\begin{array}{l}\text { Liderazgo } \\
\text { Información } \\
\text { Tecnología }\end{array}$ & $\begin{array}{l}\text { Definir como estrategia } \\
\text { la actualización tecnológica, } \\
\text { propiciar una cultura } \\
\text { organizacional. }\end{array}$ \\
\hline $\begin{array}{l}\text { Modelo de capital } \\
\text { intelectual } \\
\text { (Drogonetti y Roos) }\end{array}$ & $\begin{array}{l}\text { El valor de la compañía proviene } \\
\text { de sus activos físicos y monetarios } \\
\text { (capital financiero) y de sus recursos } \\
\text { intangibles (capital intelectual). Clasificar } \\
\text { bajo el nombre de capital intelectual } \\
\text { todos los recursos intangibles y analizar } \\
\text { sus interconexiones. Dentro del capital } \\
\text { intelectual hay dos categorías generales, } \\
\text { capital humano y capital estructural. }\end{array}$ & $\begin{array}{l}\text { Recursos financieros } \\
\text { Capital humano } \\
\text { (intangibles) } \\
\text { Partes interesadas } \\
\text { Equipos de trabajo }\end{array}$ & $\begin{array}{l}\text { La organización debe identificar } \\
\text { las necesidades financieras } \\
\text { y proveer los recursos para } \\
\text { el desarrollo de operaciones } \\
\text { que permitan procesos innovadores } \\
\text { en donde se genere y transfiera } \\
\text { el conocimiento. } \\
\text { Estimular a las personas para } \\
\text { el trabajo orientado a la mejora } \\
\text { del desempeño. } \\
\text { Propiciar el aprendizaje colectivo. }\end{array}$ \\
\hline
\end{tabular}




\begin{tabular}{|c|c|c|c|}
\hline $\begin{array}{c}\text { MODELO } \\
\text { DE GESTIÓN } \\
\text { DEL CONOCIMIENTO }\end{array}$ & CARACTERÍSTICAS PRINCIPALES & $\begin{array}{c}\text { ELEMENTOS } \\
\text { QUE EMERGEN } \\
\text { PROPIOS } \\
\text { DE LA GESTIÓN } \\
\text { DE LA CALIDAD }\end{array}$ & $\begin{array}{c}\text { ESTRATEGIA } \\
\text { DE LA ORGANIZACIÓN }\end{array}$ \\
\hline Modelo Arthur Andersen & $\begin{array}{l}\text { Responsabilidad personal de compartir } \\
\text { y hacer explícito el conocimiento } \\
\text { para la organización. Responsabilidad } \\
\text { organizacional de crear la infraestructura } \\
\text { de soporte para que la perspectiva } \\
\text { individual sea efectiva, creando los } \\
\text { procesos, la cultura, la tecnología } \\
\text { y los sistemas que permitan capturar, } \\
\text { analizar, sintetizar, aplicar, valorar } \\
\text { y distribuir el conocimiento. }\end{array}$ & $\begin{array}{l}\text { Gestión por procesos } \\
\text { Estructura documental } \\
\text { Cultura organizacional } \\
\text { Ambiente de trabajo } \\
\text { Sistemas } \\
\text { de información } \\
\text { Innovación }\end{array}$ & $\begin{array}{l}\text { Adoptar un enfoque basado } \\
\text { en procesos. } \\
\text { Crear un sistema documental } \\
\text { que permita preservar, mantener } \\
\text { y transmitir el conocimiento. } \\
\text { Crear ambientes de trabajo } \\
\text { que fomenten la productividad, } \\
\text { creatividad y bienestar de las } \\
\text { personas que en ella permanecen. } \\
\text { Se deben crear mecanismos para } \\
\text { recopilar la información requerida } \\
\text { en la gestión organizacional. } \\
\text { Diseñar sistemas de información } \\
\text { y comunicación seguros, } \\
\text { integrales y confidenciales. }\end{array}$ \\
\hline $\begin{array}{l}\text { Navigator de Skandia } \\
\text { (Edvinsson y Malone). } \\
\text { Los elementos del capital } \\
\text { intelectual son: }\end{array}$ & $\begin{array}{l}\text { Capital humano: conocimientos, } \\
\text { habilidades, actitudes de las personas } \\
\text { que componen la organización. } \\
\text { Capital estructural: conocimientos } \\
\text { explicitados por la organización. } \\
\text { Integrado por tres elementos: } \\
\text { - Clientes: activos relacionados con } \\
\text { los clientes (fidelización, capacidad } \\
\text { de conformar equipos mixtos). } \\
\text { - Procesos: forma en que la empresa } \\
\text { añade valor a través de las diferentes } \\
\text { actividades que desarrolla. } \\
\text { - Capacidad de innovación: posibilidad } \\
\text { de mantener el éxito de la empresa } \\
\text { en el largo plazo a través del desarrollo } \\
\text { de nuevos productos o servicios. }\end{array}$ & $\begin{array}{l}\text { Gestión } \\
\text { de las personas } \\
\text { Clientes y partes } \\
\text { interesadas } \\
\text { Procesos } \\
\text { Innovación }\end{array}$ & $\begin{array}{l}\text { Desarrollar las competencias } \\
\text { de los trabajadores. } \\
\text { Conformar equipos } \\
\text { interdisciplinarios de trabajo } \\
\text { en donde se socialice y } \\
\text { divulgue el conocimiento. } \\
\text { Se debe adoptar el enfoque } \\
\text { basado en procesos, crear } \\
\text { un sistema documental. } \\
\text { Incluir la innovación dentro } \\
\text { de sus políticas organizacionales } \\
\text { y estrategias de éxito. } \\
\text { Potenciar el capital humano } \\
\text { y estructural, para estimular } \\
\text { la innovación continua. } \\
\text { Se debe tener un mayor } \\
\text { conocimiento del cliente, aprender } \\
\text { de sus necesidades y expectativas } \\
\text { para generar mayor conocimiento. }\end{array}$ \\
\hline
\end{tabular}

A partir de los elementos analizados, se plantea el esquema de la figura 1 a fin de buscar una articulación de aquellos, en aras de integrar los aspectos considerados clave para que Districarnazas Luna, desde su modelo de calidad, direccione sus esfuerzos hacia la mejora de sus procesos, en donde el conocimiento se convierta en el eje dinamizador, se genere un mayor aprendizaje personal y organizacional que redunde en una significativa ventaja competitiva, un mejor posicionamiento dentro de su sector y un nivel de madurez más elevado que permita que el flujo de conocimiento sea continuo. 
Figura 1. Esquema propuesto de integración de un modelo de gestión de calidad y un modelo de gestión de conocimiento

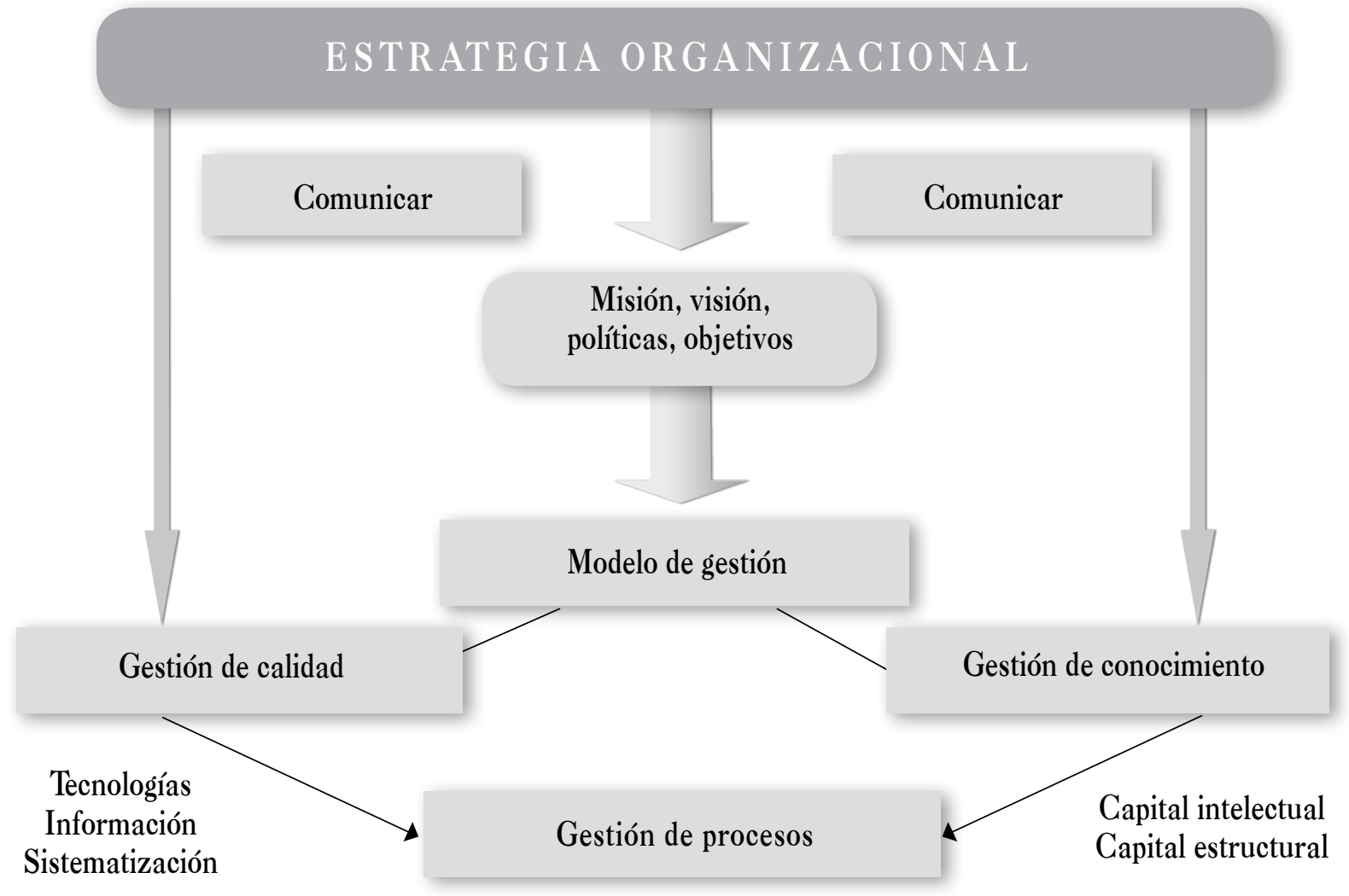

\section{GESTIÓN DE PERSONAS GESTIÓN DE INFORMACIÓN GESTIÓN DE RECURSOS}

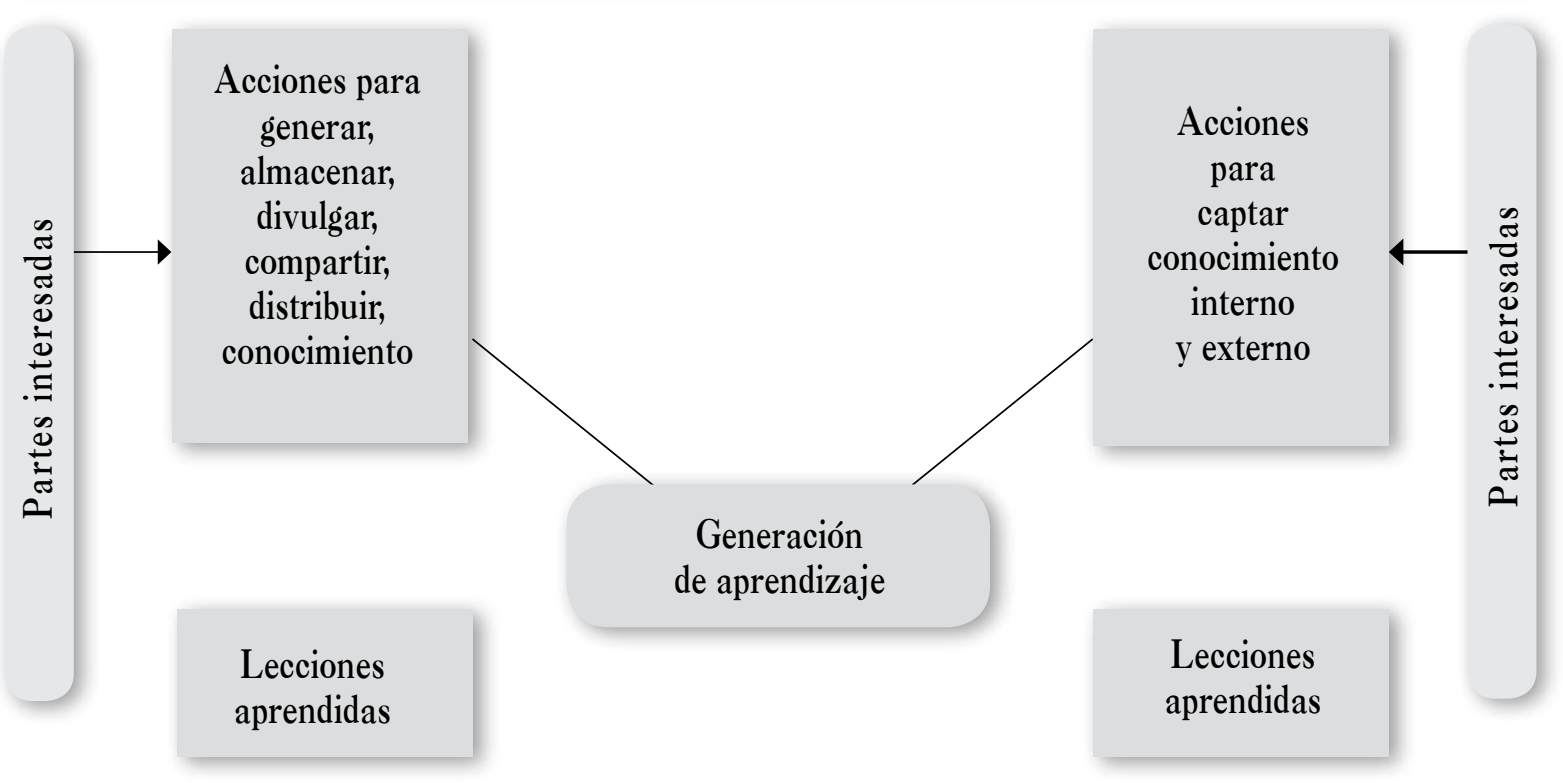

Éxito sostenido 
La estrategia de la organización debe contemplar, en primer lugar, la definición clara de su misión, visión, políticas y objetivos. A partir de esto, ha de comunicarlos a sus trabajadores por medio de diferentes mecanismos que garanticen que las personas los interioricen y se involucren en su cumplimiento; esta es la base para sustentar un modelo de gestión. Desde aquí se clarifican sus propias necesidades y se direcciona a la compañía al logro de sus estrategias corporativas. Este proceso requiere una revisión continua teniendo en cuenta que los mercados no son estáticos, por lo cual se inicia un ejercicio dinámico de renovación del conocimiento.

Para operativizar este esquema, es necesario determinar un ciclo para que la GC sea parte de la dinámica empresarial de Districarnazas Luna.

Figura 2. Operativización del esquema propuesto

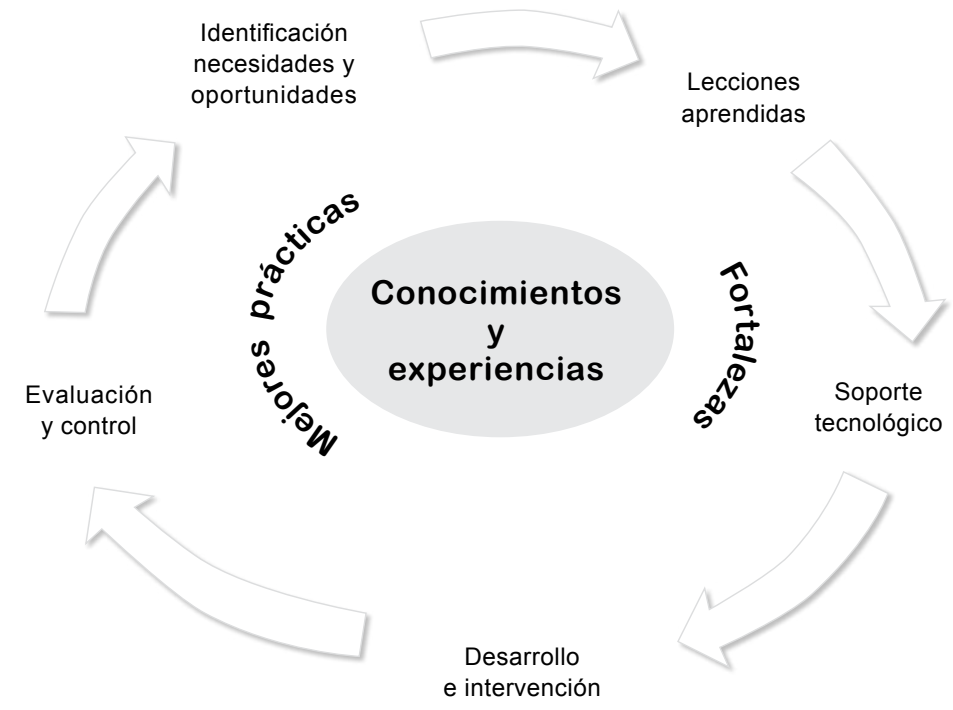

En esta figura se muestra cómo, a partir de la identificación de necesidades, las experiencias y solución de problemas se convierten en lecciones aprendidas y se capitalizan como acciones o prácticas por parte de los trabajadores y, a su vez, se reflejan como fortalezas de la organización. Es preciso que estas acciones estén debidamente documentadas y se difundan; por esta razón se requiere un soporte tecnológico que permita no solo el almacenamiento, sino la divulgación, el acceso oportuno a la información y su actualización permanente. Para ello se hace importante el uso de tecnologías de la información y de la comunicación que permitan cumplir con ese objetivo. El ciclo continúa con el desarrollo e intervención, en términos del despliegue que requiere validar la efectividad del recurso tecnológico, lo cual implica un seguimiento para realizar los ajustes del caso.

Se hace necesario desarrollar un plan de formación, a partir del cual se posibilite el flujo libre de las ideas, experiencias y conocimientos. Para tal propósito, se debe definir un plan de acción que recoja el aprendizaje de los individuos.

Para que esto se convierta en un esquema integrador, se precisa involucrar a las partes interesadas dado que son las personas quienes poseen el conocimiento; se requiere de la voz del cliente para conocer sus necesidades y expectativas, con ello se diseñan y producen los bienes y servicios a la medida, con el nivel de calidad requerido, es decir, se establecen especificaciones técnicas. Los trabajadores integran sus conocimientos, producen ideas que ayudan en la mejora del proceso y, en consecuencia, del producto. Los proveedores aportan su conocimiento en la búsqueda de mejores soluciones que favorezcan mutuamente a las dos partes. Por otro lado, el conocimiento de la competencia le permite a la organización generar nuevos y mejores desarrollos. La comunidad precisa los impactos negativos que resulten del proceso, tanto en el medio ambiente como en ella misma, de tal manera que recogiendo esta información se obtenga nuevo conocimiento que propicie el cambio de tecnologías contaminantes por tecnologías limpias y procesos que minimicen esos impactos a la comunidad y al medio, teniendo en cuenta que uno de los procesos más contaminantes es el curtido del cuero.

A continuación, se describe la manera para viabilizar la gestión del conocimiento en Districarnazas Luna, a partir del mapa de conocimiento propuesto (figura 3). 


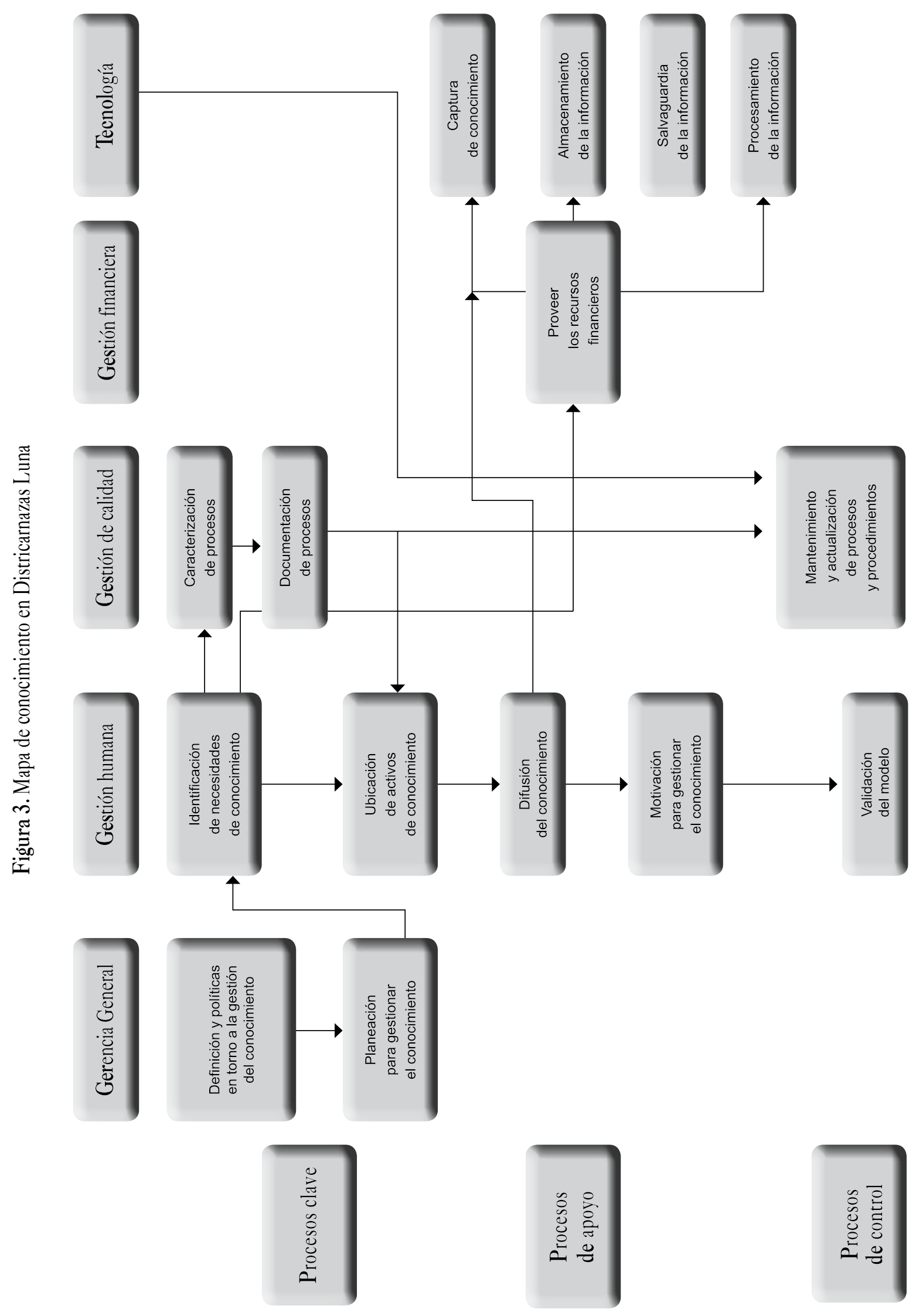


En una organización que cuenta con un SGC, es evidente que el conocimiento se plasma en los mapas de procesos, manuales de procedimientos, planes, programas e instructivos; sin embargo, para que el conocimiento se transmita, perdure, almacene y divulgue, integrándolo dentro del sistema de calidad, se debe dar el direccionamiento estratégico para facilitar la creación de conocimiento.

El mapa del conocimiento propicia interacción de las diferentes áreas de la empresa Districarnazas Luna, y permite identificar necesidades y oportunidades de conocimiento, ubicar los activos de conocimiento, estructurando la organización en torno a la gestión del conocimiento.

Es preciso definir unas entradas y unas salidas que le den solidez a la propuesta; estas son:

Entradas internas: Liderazgo, conocimiento tácito y explícito, soporte tecnológico, cultura organizacional, sistema de información, estructura corporativa, entre otras.

Entradas externas: Es importante recoger la voz del cliente, sus necesidades y expectativas, la experiencia de los competidores y los desarrollos tecnológicos que le provean a la organización herramientas para hacer más dinámica la transmisión y generación de conocimiento.

Salidas: Información, documentación, programas de divulgación y capacitación.

La empresa debe disponer de las herramientas tecnológicas adecuadas para soportar los procesos que ayuden en la toma de decisiones y sean un canal de comunicación continuo entre los trabajadores, y apoyen la salvaguardia y difusión del conocimiento, por esto se hace indispensable tener una política de actualización continua.

\section{CONCLUSIONES}

A partir de la información obtenida, luego de aplicar el instrumento de investigación diligenciado con los trabajadores y directivos de la empresa Districarnazas Luna y su contraste con la teoría, se presentan y analizan los resultados obtenidos.

Se evidencia en la organización una administración mecanicista, más orientada a los resultados de carácter comercial y económico, en donde los colaboradores participan con su conocimiento, creatividad e ideas para aportar a la empresa, pero esto se da más por un proceso inercial y no como resultado de una orientación directiva. Aunque si bien es cierto que hay unos rasgos que hablan de mecanismos de difusión de conocimiento, estos obedecen a la estructura del sistema de calidad que se implementa por la necesidad de participar en las licitaciones con el Estado, pero que de alguna manera le permitieron a la empresa organizar y documentar sus procesos.

En cuanto a la encuesta a jefes y directivos, se apreció que a pesar de que en la empresa Districarnazas Luna existen aspectos que se valoran del conocimiento de los trabajadores, estos no obedecen a lineamientos ni directrices claras en torno a la gestión del conocimiento y a las acciones derivadas del modelo de calidad frente a este tema, que obligan al cumplimiento de unos requisitos de la norma, no trascienden en la política y los objetivos. Por ende, de cara al abordaje del capital intelectual de trabajador, este no llega a ser un aspecto diferenciador frente a las nuevas realidades que mueven el mundo de los negocios, en el cual la calidad debe ir de la mano del conocimiento de los individuos, y viceversa, y que marca una ventaja competitiva en las organizaciones.

Es de resaltar que la empresa se caracteriza por ser líder en el mercado del cuero. Ha construido un 
acervo importante de conocimiento, el cual se usa dentro de su proceso productivo, ha incorporado nuevas tecnologías y ha expandido su negocio de manera significativa; sin embargo, esto no responde a una dinámica de construcción y transformación de conocimiento de manera estratégica, pues se da a partir de las mismas necesidades del negocio, por exigencias del mercado, y no como consecuencia de los planes estratégicos por mantenerse en una posición de ventaja competitiva.

A partir de las encuestas aplicadas a jefes, directivos y trabajadores de base, se concluye que la empresa tiene un nivel de madurez organizativa bajo en lo que respecta a la gestión del conocimiento, dado que no cuenta con la suficiente capacidad para movilizar la asimilación y realización de prácticas encaminadas a generar una cultura de conocimiento en la compañía que apoyen una gestión decidida del conocimiento.

Frente a la madurez tecnológica, se pudo observar que aunque Districarnazas Luna ha incorporado tecnologías de información para la mejora del proceso administrativo, software de apoyo, internet e intranet, importantes para la ejecución de tareas, no se convierten en el elemento dinamizador para transferir y crear nuevo conocimiento.

En lo que respecta al sistema de gestión de la calidad, este tiene un grado de importancia relativa, en virtud a que desde el momento de su implementación imprimió cambios importantes en la manera de realizar las funciones propias de la empresa. En tanto tareas, actividades y procesos han logrado una mejora, con lo cual la organización consiguió resultados importantes en el mercado. A pesar de esto, el SGC no ha permitido fluir el conocimiento organizacional ni de las personas, ya que se le ha dado poco valor al sistema documental y a las prácticas que se deben fomentar para propiciar la generación de ideas y el aprendizaje continuo, incluyendo el aprender de los propios errores.

Confrontado lo que Districarnazas Luna tiene dentro del modelo de gestión, se tiene claro que el conocimiento se representa en la experiencia, estudio del mercado, su marca y en sus trabajadores. Esto es lo que le permite a esta organización ser competitiva y tener reconocimiento en el sector del cuero y derivados.

Por lo visto anteriormente, se puede concluir que, frente a la gestión del conocimiento, Districarnazas Luna se encuentra en un nivel básico (bajo) de madurez frente al tema, dado que posee poca experiencia implementando modelos de gestión del conocimiento, no están definidas unas directrices y líneas de acción consistentes con sus estrategias corporativas, se limitan la acción y participación del trabajador y no se crea el ambiente propicio para compartir ideas, los canales de comunicación existentes no son eficientes.

El panorama expuesto visibiliza los retos que la implementación de sistemas de gestión de conocimiento impone a las organizaciones de cara a la transformación y recontextualización permanente del saber y la información. Esto exige cambios profundos en las maneras de concebir, gerenciar y administrar el talento humano, cambios que pasan por el reconocimiento de los trabajadores y han de perseguir una sinfonía empresarial en busca de propósitos similares. Así, a pesar de contar con sistemas de gestión de calidad (SG), es esencial que Districarnazas Luna vuelva la mirada a las personas como artífices de ideas que, decantadas, estimuladas y comunicadas, redunden en el logro de los objetivos misionales.

Con esta investigación se espera aportar a la discusión académica, en particular a la Maestría en Calidad y Gestión Integral, a fin de obtener un documento orientador que facilite el camino a la dinamización e integración de los diferentes sistemas de gestión. 


\section{RECOMENDACIONES}

Se propone un proceso de sensibilización e interiorización del cuerpo directivo de Districarnazas Luna, a fin de que tenga los elementos que propicien una integración del SGG con un modelo de GC; por ello se hace importante recomendar el diseño de herramientas que integren en uno solo los modelos de gestión.

\section{REFERENCIAS BIBLIOGRÁFICAS}

BARRIO FERNÁNDEZ, Norma M. y SOTO BALBÓN, María Aurora. Gestión del conocimiento. Parte I: revisión crítica del estado del arte. En: Acimed. 2006. vol. 14, no. 2, p. 1-36.

BUENO, Eduardo. Enfoques principales y tendencias en dirección del conocimiento. En: Gestión del conocimiento: desarrollos teóricos y aplicaciones. 2003, p. 21-54

CAMISÓN César; CAMISÓN ZORNOZA César; GONZÁLEZ CRUZ Tomás; CRUZ Sonia y GONZÁLEZ, Tomás. Gestión de la calidad: concepto, enfoques, modelos y sistemas. Madrid: Pearson Prentice Hall, 2007. 105 p.

CHIAVENATO, Idalberto. Gestión del talento humano. 1 ed. México: McGraw-Hill, 2004.

ORTIZ DE URBINA, Marta. Medición y auditoría del capital intelectual. En: El profesional de la información. Julio, 2003. vol. 12, no. 4, p. 282-289.

VÁZQUEZ RECIO, Rosa y ANGULO RASCO, Félix. Introducción a los estudios de caso. Archidona, España: Ediciones Aljibe, 2003. 232 p.

\section{Webgrafía}

ANDERSEN, Arthur. Modelo Andersen [en línea], 1999 [citado en 12/01/2013]. Disponible en Internet: <http://www.gestiondelconocimiento.com/ modelos_arthur.htm>

BERNUY, Augusto Ernesto. Gestión colaborativa del conocimiento [en línea], [citado en 20/01/2013]. Disponible en Internet: <http://eventos.spc.org. pe/cicp2005/papers/0013/Paper\%20III\%20ABernuy\%20rmcpperu2005\%20-\%20Gestion\%20Colaborativa\%20del.pdf>

BUENO, E. Modelo de dirección estratégica por competencias: el capital intangible [en línea], 1998 [citado en 2/02/2013]. Disponible en Internet: $<$ http://www.gestiondelconocimiento.com/modelos_bueno.htm>

CARABALLO, Yeter, MESA, Dianelis y HERRERA, J. A. Herramientas de gestión del conocimiento: convergencias hacia un aprendizaje organizacional. En: Revista Cubana de Ciencias Agrícolas. 2009. vol. 43, no. 1.

CLUB DE GESTIÓN DEL CONOCIMIENTO Y LA INNOVACIÓN DE LA COMUNIDAD VALENCIANA. Modelo Nova [en línea], 1999 [citado en 11/02/2013]. Disponible en Internet: <http:// www.gestiondelconocimiento.com/modelo_valencia.htm>

DELGADO VERDE, Miriam, LOPÉZ SÁENZ, Pedro, NAVAS, José y MARTÍN, Gregorio. Propuesta de un modelo sobre dinámicas de aprendizaje organizativo [en línea], [citado en 15/01/2013]. Disponible en Internet: <http://www.madrimasd. org/revista/revista44/aula/aula1.asp> 
DRAGONETTI, N. C.; ROSS, G., Capital Intelectual [en línea], 1998 [citado en 18/01/2013]. Disponible en Internet: <http://www.gestiondelconocimiento.com/modelos_capital_intelectual.htm>

EDVINSSON, Knowledge Management Assessment Tool (Kmat) [en línea], [citado en 05/02/2013]. Disponible en Internet: <http://www.gestiondelconocimiento.com/modelos_kmat.htm>

EUROFORUM. Modelo Intelec [en línea], [citado en 13/01/2013]. Disponible en Internet: <http:// www.gestiondelconocimiento.com/modelo_ modelo_intelect.htm>

LÓPEZ G., María del Socorro; CABRALES G, Fernando y SCHMAL S., Rodolfo. Gestión del conocimiento: una revisión teórica y su asociación con la universidad. [Chile]: Red Panorama Socioeconómico, 2005 [citado en 23/02/2013]. Disponible en Internet: <http://site.ebrary.com/lib/bibliotecaustasp/Doc?id=10102738\&ppg=4>

PÉREZ PÉREZ, Mario Frank. La gestión del conocimiento y la cultura organizacional [en línea], [citado en 25/01/2013]. Disponible en Internet: <http://cadenahortofruticola.org/admin/tecno/176gestion_ conocimiento_upb.pd>

SVEIBY. Intellectual Assets Monitor [en línea], 1997 [citado en 17/01/2013]. Disponible en Internet: $<$ http://www.gestiondelconocimiento.com/modelos_sveiby.htm>

TEJEDOR, B., \& AGUIRRE, A. (1998). Modelo de Gestión del Conocimiento de KPMG Consulting. [en línea], [citado en 06/02/2013]. Disponible en Internet: <http://www.gestiondelconocimiento.com/ modelos_kpmg.htm> 